\section{Postoperative Course}

The patient's condition stabilized, and sedation was discontinued the first postoperative day. The patient was free of inotropic support on day 3 and was successfully extubated. He showed no neurologic abnormalities and no rhythm disorders. The patient was discharged 20 days postoperatively, and he was alive and well (New York Heart Association functional class II) on postoperative day 61 .

\section{DISCUSSION}

Transcatheter aortic valve implantation as a treatment option for severe aortic valve stenosis has been a major development in cardiovascular medicine of the last 5 years and has become a routine procedure at many hospitals. Recent studies have shown encouraging results, especially considering the high-risk population that has been treated. ${ }^{1,2}$ Concomitant treatment of coronary artery disease by percutaneous intervention has been described. ${ }^{3}$ In addition, single cases or small series of transcatheter mitral valve-invalve procedures, as well as 1 double valve procedure, have been reported. ${ }^{4,5}$ The combination of all these procedures, however, has never before been successfully performed. The case presented here was thoroughly discussed by a heart team after conventional surgery was denied. All involved persons, including the patient, were aware of the fact that this hybrid approach could fail at any step of the procedure. It was, however, considered the least risky option for the patient. There was also general agreement that treating only some of the problems did not have sufficient potential for symptom relief and might on the contrary present a risk for slow postoperative recovery.

The procedural planning and conduct of this heart team provides a good example of how treatment options can be expanded for patients with a very high operative risk by good collaboration between cardiologists and cardiac surgeons.

\section{References}

1. Leon MB, Smith CR, Mack M, Miller DC, Moses JW, Svensson LG, et al. Transcatheter aortic-valve implantation for aortic stenosis in patients who cannot undergo surgery. N Engl J Med. 2010;363:1597-607.

2. Reynolds MR, Magnuson EA, Lei Y, Leon MB, Smith CR, Svensson LG, et al. Health-related quality of life after transcatheter aortic valve replacement in inoperable patients with severe aortic stenosis. Circulation. 2011;124:1964-72.

3. Mostafa AE, Geist V, Abdel-Wahab M. Ad-hoc percutaneous coronary intervention and transcatheter aortic valve implantation as a combined transfemoral procedure. J Invasive Cardiol. 2011;23:E102-5.

4. Cheung AW, Gurvitch R, Ye J, Wood D, Lichtenstein SV, Thompson C, et al. Transcatheter transapical mitral valve-in-valve implantations for a failed bioprosthesis: a case series. J Thorac Cardiovasc Surg. 2011;141:711-5.

5. Seiffert M, Baldus S, Conradi L, Koschyk D, Schirmer J, Meinertz T, et al. Simultaneous transcatheter aortic and mitral valve-in-valve implantation in a patient with degenerated bioprostheses and high surgical risk. Thorac Cardiovasc Surg. 2011;59:490-2

\title{
Treatment of large subglottic tracheal schwannoma with microdebrider bronchoscopy
}

\author{
Jose Melendez, MD, ${ }^{\mathrm{a}}$ Lorraine Cornwell, MD, ${ }^{\mathrm{b}}$ Linda Green, $\mathrm{MD},{ }^{\mathrm{c}}$ and Roberto F. Casal, MD, ${ }^{\mathrm{a}}$ Houston, \\ Tex
}

Primary tracheal schwannomas are among the least common tracheal tumors. ${ }^{1}$ Although endoscopic treatment with the Nd:YAG laser has been reported, surgical resection remains the standard of care.,3 We report here the successful use of a novel bronchoscopic technique, the

\footnotetext{
From the Departments of Pulmonary and Critical Care Medicine, ${ }^{a}$ Cardiothoracic Surgery, ${ }^{\mathrm{b}}$ and Pathology and Immunology, ${ }^{\mathrm{c}}$ Michael E. DeBakey VA Medical Center, Baylor College of Medicine, Houston, Tex.

Disclosures: Authors have nothing to disclose with regard to commercial support.

Received for publication Nov 2, 2011; revisions received Dec 13, 2011; accepted for publication Jan 25, 2012; available ahead of print Feb 20, 2012.

Address for reprints: Roberto F. Casal, MD, Department of Pulmonary and Critical Care Medicine, Baylor College of Medicine, Michael E. DeBakey VA Medical Center, 2002 Holcombe Blvd, Pulmonary Section 111i. Houston, TX 77030 (E-mail: casal@bcm.edu).

J Thorac Cardiovasc Surg 2012;144:510-12

0022-5223/\$36.00

Copyright (C) 2012 by The American Association for Thoracic Surgery doi:10.1016/j.jtcvs.2012.01.072
}

microdebrider, for the management of a large subglottic tracheal schwannoma.

\section{CLINICAL SUMMARY}

A 63-year-old man was referred to our institution for diagnosis and management of a large endotracheal mass detected in a computed tomographic scan of the chest during evaluation for dyspnea. We performed rigid bronchoscopy, finding a mass on the posterior wall of the trachea, $2 \mathrm{~cm}$ from the vocal cords, with approximately $90 \%$ obstruction of the lumen (Figure 1, A). This mass was sessile, round, and broad based. Endobronchial ultrasonography was used to assess the vascularity of the lesion and its relationship to the esophagus, and to obtain a needle aspiration specimen for preliminary diagnosis. On-site cytologic examination showed a spindle cell tumor. The patient was considered to be a poor surgical candidate 


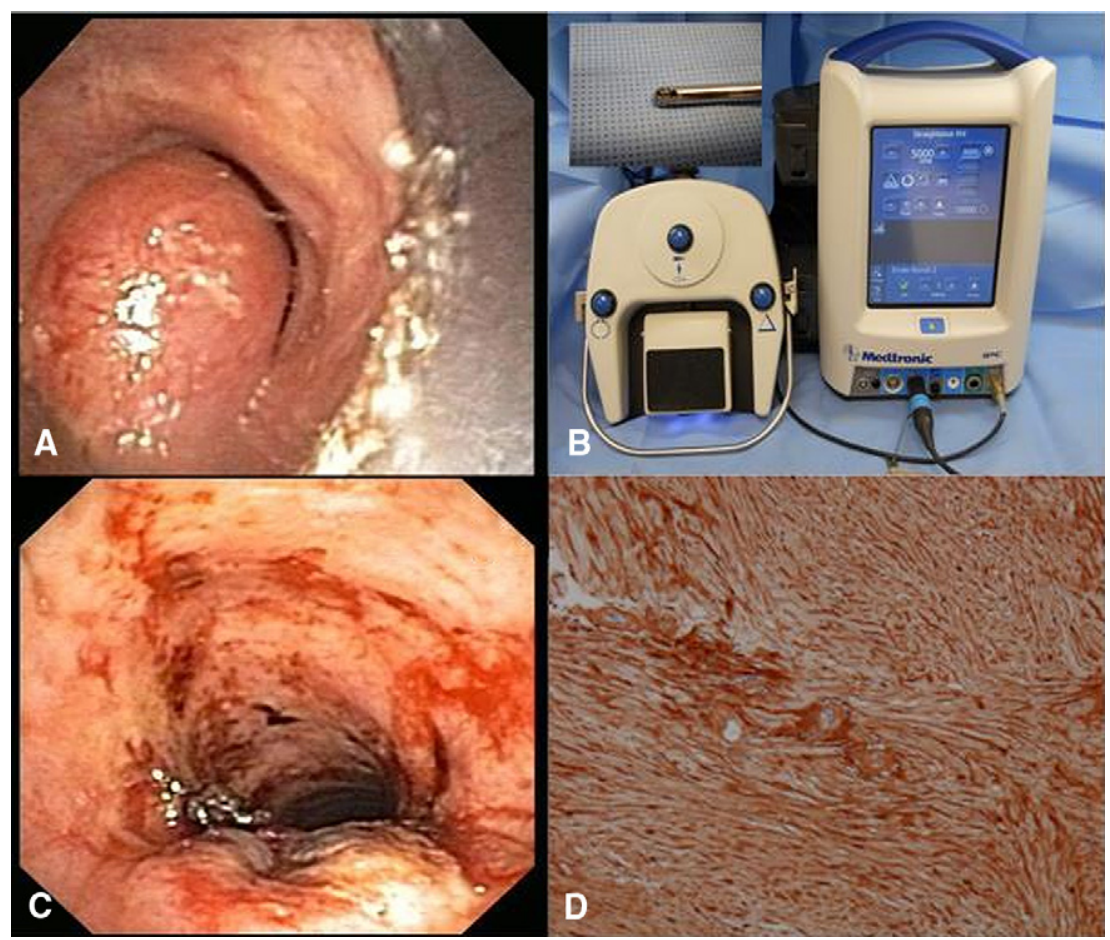

FIGURE 1. A, Large subglottic tracheal schwannoma obstructing $90 \%$ of the lumen. B, Straightshot M4 Microdebrider, powered by the Integrated Power Console (Medtronic, Inc, Minneapolis, Minn). C, Subglottic trachea after tumor debulking, showing no residual obstruction. D, Histopathologic examination, showing spindle cells staining strongly and diffusely positive for S-100, compatible with schwannoma.

because of both comorbidities and the immediately subglottic location of the lesion. Because ventilation and oxygenation were suboptimal, we decided to debulk the tumor with the Straightshot M4 Microdebrider, powered by the Integrated Power Console (Medtronic, Inc, Minneapolis, Minn; Figure 1, B). Bleeding was minimal, and there was no residual obstruction (Figure 1,C). The patient was successfully extubated, and his dyspnea was resolved.

Within a few hours after the procedure, the patient had trouble urinating and performed a prolonged Valsalva maneuver, leading to mild pneumomediastinum. This pneumomediastinum resolved spontaneously within 5 days, and the patient was discharged. Immunohistochemical staining of biopsy specimens was strongly and diffusely positive for $\mathrm{S}-100$, and the final diagnosis was benign schwannoma (Figure 1,D). Follow-up at 3 and 6 months with chest computed tomography and bronchoscopy showed no evidence of tumor recurrence, and the patient has remained free of symptoms.

\section{DISCUSSION}

Although the standard management of benign tracheal schwannomas is circumferential resection with tracheal reconstruction, bronchoscopic laser resection has also been described. ${ }^{2,3}$ In our case, because of the large size of his tumor and mild difficulty with ventilation and oxygenation during bronchoscopy, we chose to use microdebrider bronchoscopy to recanalize the airway swiftly. The microdebrider is a powered instrument composed of a hollow metal tube with a rotating blade coupled with suction that needs to be passed through a rigid bronchoscope or tracheoscope. ${ }^{4}$ The metal tube is $45 \mathrm{~cm}$ in length and $4 \mathrm{~mm}$ in diameter, with either a straight or an angled tip $\left(15^{\circ}\right)$. The handle of the microdebrider has a flywheel that allows $360^{\circ}$ rotation of the tip. After setting the rotation on the power source console, a foot pedal is used to activate the blade. The rotation is typically set at a rate of 1500 to $5000 \mathrm{rpm}$. The rotating blade of the microdebrider should be gently placed in contact with the tumor, allowing suction to bring the tumor into it. The microdebrider can reach lesions in the trachea, main stem bronchi, and bronchus intermedius. Unlike with electrocautery techniques, lowering of inspired oxygen fraction and interruptions in jet ventilation to avoid endobronchial fire are not necessary. Additionally, the microdebrider does not need to be removed and reintroduced for tumor debulking like most other bronchoscopic tools (forceps, electrocautery probes), potentially shortening the procedure time.

Although the microdebrider is a common tool for head and neck specialists, its use in bronchoscopy is relatively new, and data on effectiveness and safety are scant. ${ }^{4,5}$ Lunn and coworkers ${ }^{4}$ retrospectively described their 
successful and complication-free use of the microdebrider in 23 patients with tracheal granulation tissue, idiopathic subglottic stenosis, and malignant disease. There are no available data regarding potential complications. Because no hemostasis is provided with this technique, bleeding is always a concern. Bleeding is generally mild, however, and the suction of the microdebrider is strong enough to keep the field clear. When bleeding is significant, a suction catheter can be simultaneously introduced, or intermittent electrocautery can be performed. Extra care is required when debulking tumors arising in the posterior wall of the trachea or main bronchi because of the risk of perforation.

The cost of the equipment is largely comparable to that of other bronchoscopic tools used for tumor debulking. The use of the microdebrider is intuitively obvious, and in our opinion little training is needed for a thoracic surgeon or pulmonologist already experienced in rigid bronchoscopy.

\section{CONCLUSIONS}

The microdebrider could become a valuable alternative for the bronchoscopic management of tumors in the central airways, particularly in cases of severe airway obstruction causing suboptimal ventilation and oxygenation. Further studies are needed to establish its safety profile.

\section{References}

1. Righini CA, Lequeux T, Laverierre MH, Reyt E. Primary tracheal schwannoma: one case report and a literature review. Eur Arch Otorhinolaryngol. 2005;262: 157-60.

2. Dorfman J, Jamison BM, Morin JE. Primary tracheal schwannoma. Ann Thorac Surg. 2000;69:280-1.

3. Rusch VW, Schmidt RA. Tracheal schwannoma: management by endoscopic laser resection. Thorax. 1994;49:85-6.

4. Lunn W, et al. Microdebrider bronchoscopy: a new tool for the interventional bronchoscopist. Ann Thorac Surg. 2005;80:1485-8.

5. Kennedy MP, Morice RC, Jimenez CA, Eapen GA. Treatment of bronchial airway obstruction using a rotating tip microdebrider: a case report. J Cardiothorac Surg. 2007;2:16.

\title{
A new technique for partial removal of the pulmonary artery in video-assisted thoracic surgical lobectomy
}

\author{
Zhi Zhang, MD, Jianfeng Huang, MD, PhD, Rong Yin, MD, PhD, and Lin Xu, MD, PhD, Nanjing, China
}

Lobectomy with video-assisted thoracic surgery (VATS) is now popular worldwide and widely applied to patients with early stage non-small cell lung cancer. Lobectomy with pulmonary artery (PA) reconstruction by VATS currently remains a challenge for thoracic surgeons when the PA is invaded by tumor or lymph nodes. In traditional open thoracotomy, the surgical technique of clamping the proximal PA and the pulmonary vein (PV) of the reserved lobe is usually applied for PA reconstruction. ${ }^{1}$ We transplanted this idea into VATS and developed a new technique for partial removal and reconstruction of the PA in VATS lobectomy.

\section{TECHNIQUE}

Ethical approval for trial of VATS lobectomy with partial removal and direct linear suture of the PA was obtained

\footnotetext{
From the Department of Thoracic Surgery, Nanjing Medical University Affiliated Cancer Hospital, Cancer Institute of Jiangsu Province, Nanjing, China. Disclosures: Authors have nothing to disclose with regard to commercial support. Received for publication Oct 7, 2011; revisions received Dec 13, 2011; accepted for publication Jan 25, 2012; available ahead of print Feb 20, 2012.

Address for reprints: Lin Xu, MD, PhD, Department of Thoracic Surgery, Nanjing Medical University Affiliated Cancer Hospital, Cancer Institute of Jiangsu Province, 42 Baiziting, Nanjing, 210009, PR China (E-mail: xulin_83@yahoo.cn).

J Thorac Cardiovasc Surg 2012;144:512-4

0022-5223/ $\$ 36.00$

Copyright (c) 2012 by The American Association for Thoracic Surgery doi:10.1016/j.jtcvs.2012.01.075
}

from the institutional review board of the hospital. Between December 2007 and August 2011, a total of 5 patients agreed to undergo this procedure.

We placed an anterolateral minithoracotomy $(40 \mathrm{~mm})$ in the fourth intercostal space. An access incision $(12 \mathrm{~mm})$ was added for clamping of the PA in the third intercostal space on the anterior-axillary line. Another 2 thoracoscopic ports were placed in the seventh intercostal space on the posterior-axillary line and the midaxillary line (Figure 1, A). After the hilar dissection had been performed as previously reported, ${ }^{2}$ the PV and PA branches without cancer invasion were stapled with vascular staplers. After the superior PV had been divided to allow better viewing of the PA, the proximal PA was controlled with a Satinsky clamp. Specifically, on the right side the proximal control was obtained anterior to the superior vena cava after division of the truncus anterior of the PA; on the left side, it was necessary to transect the ligamentum arteriosum to clamp the PA trunk. A clamp was placed through the incision (third intercostal space) on the proximal PA after intravenous injection of $3125 \mathrm{U}$ sodium heparin. Rather than clamping the PV of the reserved lobe to avoid backflow, ${ }^{2}$ an umbilical tape was used to block the reserved PV and was secured with a 5-mm Hem-o-lok clip (Teleflex Medical Inc, Durham, NC), which can provide more space for the PA reconstruction. The PA branches with cancer invasion were 